\title{
Desempeño de una prueba rápida para el diagnóstico de sífilis en mujeres puérperas
}

\author{
Verónica Villagra ${ }^{\mathrm{I}}$, Mauricio Goldman ${ }^{\mathrm{I}}$, María Liz Bobadilla ${ }^{\mathrm{I}}$, Gladys Olmedo ${ }^{\mathrm{I}}$, Margarita Cabral ${ }^{\mathrm{II}}$, Víctor \\ Alfonso ${ }^{\mathrm{I}}$ \\ I Laboratorio Central de Salud Pública. Departamento de Inmunología, Asunción - Paraguay. \\ II Universidad Católica Nuestra Señora de la Asunción. Paraguay
}

\author{
Cómo referenciar este artículo/ \\ How to reference this article:
}

\author{
Villagra V, Goldman M, Bobadilla M, Olmedo G, \\ Cabral M, Alfonso V. Desempeño de una prueba \\ rápida para el diagnóstico de sífilis en mujeres \\ puérperas. Mem. Inst. Investig. Cienc. Salud. \\ 2016;14(3):115-120
}

\section{RE S U M E N}

La sífilis, por su impacto en la salud materno infantil, demanda prevención y tratamiento de calidad. Las pruebas rápidas son herramientas adecuadas de detección con una sensibilidad entre $84 \%$ a $97 \%$ y especificidad de $84 \%$ o más. En Paraguay, las pruebas rápidas han sido validadas e incorporadas a los programas de salud pero no existen estudios que hayan evaluado su desempeño, por lo que este estudio observacional descriptivo tiene por objetivo evaluar el desempeño de una prueba rápida comercial para el diagnóstico de sífilis en comparación a la Hemaglutinación Indirecta del Treponema (TPHA) en muestras de mujeres puérperas remitidas al Laboratorio Central de Salud Pública por hospitales de parto del Municipio Capital, y los departamentos: Central, Concepción y Alto Paraná entre 2011 y 2012. De 334 muestras evaluadas, 108 fueron positivas por las dos pruebas (test rápido y TPHA), y 182 negativas por ambas. En el resto, los resultados fueron discordantes (23 positivos con la prueba rápida y negativos por TPHA y 21 negativos por test rápido y positivos por TPHA). La sensibilidad de la prueba rápida comparada con TPHA fue $83,72 \%$ (IC95\%: 75,96-89,42\%) y la especificidad de $88,78 \%$ (IC95\%: 83,45$92,61 \%)$.

Palabras clave:sifilis,tiras rapidas, validación

\section{Performance of a rapid test for the diagnosis of syphilis in puerperal women}

\section{A B S T R A C T}

Due to its impact on maternal and child health, syphilis demands quality in prevention and treatment. The rapid test is an appropriate screening tool with a sensitivity of $84-97 \%$ and specificity from $84 \%$. In Paraguay, rapid tests have been validated and incorporated into health programs. The performance of a rapid test for syphilis was evaluated compared with Treponema particle agglutination assay (TPHA) in samples of puerperal women sent to the Central Laboratory of Public Health from birthing hospitals of the Capital City, and three departments: Central, Concepción and Alto Paraná between 2011 and 2012. Of the 334 samples tested, 108 were positive by both tests (rapid test and TPHA test), and 182 were negative by both. The rest presented discordant results ( 23 positive with the rapid test and TPHA negative and 21 negative by rapid test and positive for TPHA). The sensitivity of the rapid test compared with the TPHA was $83.72 \%$ (95\% CI 75.96 to $89.42 \%$ ) and specificity $88.78 \%$ (95\% CI 83.45 to 92.61 ).

Keywords: Syphilis, rapid test, validation

\section{INTRODUCCIÓN}

El acceso al diagnóstico temprano en embarazadas y puérperas, mediante test rápidos reducen la morbimortalidad de esta enfermedad que puede afectar al feto en cualquier 
momento durante el embarazo y parto, siendo fundamental determinar su efectividad diagnóstica como alternativa valiosa en la implementación de programas de prevención y vigilancia del VIH y/o sífilis $(1,2)$.

El diagnóstico de la sífilis se basa en el cuadro clínico del paciente, factores epidemiológicos y métodos de laboratorio. Los métodos directos aíslan el microorganismo a través de cultivos $\mathrm{y} / \mathrm{o}$ métodos moleculares. Los indirectos pueden ser treponémicos: detectan anticuerpos antitreponémicos producidos por el individuo en respuesta al estímulo del T. pallidum; de tipo IgA, IgM o IgG, entre los cuales están: Inmunoanálisis Enzimático (ELISA), Hemaglutinación Indirecta del Treponema (TPHA), Inmunocromatografía (IC)dentro de la que se encuentran las pruebas rápidas, Absorción de Anticuerpos Treponémicos Fluorescentes (FTA-ABS), y los no treponémicas que determinan anticuerpos no treponémicos que son liberados por el tejido necrotizado de la lesión, como ser Venereal Disease Research Laboratory (VDRL), y Prueba Rápida de Reaginas Plasmáticas (RPR), Undeated Serum Reagin (USR)(3-5).

Las pruebas serológicas se vuelven reactivas pasadas 3 a 4 semanas desde el inicio de las lesiones y la sensibilidad y especificidad de las mismas varían según los diferentes estadios de evolución de la enfermedad $(1,3)$. La mayoría de estas pruebas necesitan de infraestructura de laboratorio no así la prueba rápida ya que se requiere un mínimo entrenamiento, y muy poca infraestructura. Al contar con la misma cuyo resultado se tendría en 15 minutos posteriores a la obtención de la sangre, se facilita la aplicación sistemática de la prueba diagnóstica en los diferentes niveles de atención médica (6-9).

El objetivo del estudio fue comparar el desempeño de una prueba rápida para sífilis con la Prueba TPHA como prueba de referencia utilizada para el diagnóstico de sífilis en muestras de puérperas o parturientas remitidas a Laboratorio Central por hospitales de parto del municipio capital: Hospital Materno Infantil de San Pablo, Hospital de Trinidad, del Departamento Central: Hospital Regional de Luque, Hospital Distrital de Lambaré, Hospital Distrital de Capiatá, Hospital Distrital de Mariano R. Alonso, y Centro de Salud Ypané y Hospital Regional de la X Región Sanitaria, Hospital Regional de Coronel Oviedo y de la I Región Sanitaria del Paraguay, durante el año 2011 y 2012.

\section{MATERIAL Y METODOS}

Se realizó un estudio retrospectivo de corte transversal en el que se incluyeron muestras de suero pertenecientes a mujeres puérperas de13 a 55 años remitidas al Laboratorio Central durante los años 2011 y 2012 por hospitales de parto del municipio capital; Hospital Materno Infantil de San Pablo, Hospital de Trinidad y Departamento Central; Hospital Regional de Luque, Hospital Distrital de Lambaré, Hospital Distrital de Capiatá, Hospital Distrital de Mariano R. Alonso, y Centro de salud Ypané y Hospital Regional de la X Región Sanitaria, Hospital Regional de Coronel Oviedo y de la I Región Sanitaria del Paraguay. Las muestras de suero estaban acompañadas con las correspondientes planillas de datos en Excel que fueron llenados por profesionales de la salud de los diferentes Hospitales de Parto. Fueron incluidas solo aquellas muestras que contaban con resultados de test rápido y TPHA, ya que en algunas ocasiones no se contaba con los reactivos de TPHA realizándose otras pruebas como FTA ABS, datos no analizados aquí. Se excluyeron 30 muestras (aquellas sin datos completos, muestras hemolizadas y/o lipémicas). Los centros de parto remitieron los resultados de pruebas rápidas de cada paciente.

La prueba rápida utilizada (test rápido Inmunocromatográfico) es de marca SD Diagnostics (Gyeonggi-do, Corea del Sur).La prueba SD BIOLINE Syphilis 3.0 es un ensayo Inmunocromatográfico de fase sólida para la detección cualitativa de anticuerpos de todos los isotipos (IgG, IgM, IgA) contra Treponema pallidum (TP). Un resultado era positivo cuando había presencia aunque sea débil de una banda de color en línea de control y paciente.

Las planillas de resultados fueron remitidas por los Hospitales de Parto con los datos de las puérperas como edad, código, mortinato o aborto y pruebas de rápidas efectuadas. Los datos socio demográficos fueron tomados por personal de salud en el momento de la toma de muestra en los hospitales.

Los resultados fueron confirmados en el departamento de Inmunología del Laboratorio central de Salud Pública con Líquid SÍFILIS TPHA de marca Human(Wiesbaden, Alemania), prueba de hemaglutinación indirecta para la determinación cualitativa y cuantitativa de anticuerpos en suero frente al antígeno Treponema pallidum. Los eritrocitos aviares de las placas están recubiertos con el antígeno $T$. pallidum. En presencia de anticuerpos específicos para $T$. pallidum las células de ensayo se aglutinan debido a una reacción 
antígeno-anticuerpo. Si no hay anticuerpos específicos para $T$. pallidum, las células se sedimentan rápidamente. El botón compacto en la parte inferior de los pozos indica un resultado negativo, mientras que una capa uniforme de células aglutinadas, a veces rodeadas por un anillo, indican un resultado positivo. Con las células de control se debe ver siempre un botón bien definido.

Los datos se recolectaron de las planillas de resultados remitidos por los hospitales de parto y planillas de resultados de LCSP.

Aspectos Éticos. Las muestras analizadas se manejaron por códigos y en forma confidencial.

Análisis estadístico. Los resultados introducidos en una planilla Excel (Microsoft Office, 2010) fueron analizados con herramientas de estadística descriptiva, calculándose la sensibilidad y la especificidad con sus intervalos de confianza al 95\% con EpiInfo versión 3.4.1, 2007 (Centers for Disease Control and Prevention, Atlanta, GA).

\section{RESULTADOS}

Fueron estudiadas 334 muestras de mujeres puérperas entre 13 y 55 años de edad (promedio de 27,25y desviación estándar de7,04 años).

La sensibilidad de la prueba rápida comparada con el TPHA fue de 83,72\%(IC95\%: $75,96-89,42 \%$ ) y la especificidad fue de $88,78 \%$ (IC del $95 \%: 83,45-92,61 \%$ ) mientras que los valores predictivos positivos y negativos fueron $82.44 \%$ (IC del $95 \%: 74.61 \%$ $88.32 \%$ ) y89.66\%(IC del $95 \% 84.42 \%-93.33 \%$ ) respectivamente (Tabla 1 ).

Tabla 1. Valores de Sensibilidad, (S) Especificidad (E) y Valores Predictivos Negativos y Positivos (V.P.N y V.P.P) de la prueba rápida con respecto a TPHA. $n=334$

\begin{tabular}{llll}
\hline & & \multicolumn{2}{c}{ TPHA } \\
\cline { 3 - 4 } & & Positivo & Negativo \\
\hline \multirow{2}{*}{ Test rápido } & Positivo & 108 & 23 \\
& Negativo & 21 & 182 \\
& Total & 129 & 205 \\
\hline
\end{tabular}

\begin{tabular}{llll}
\hline \multirow{2}{*}{ Parámetros } & \multicolumn{2}{c}{ Intervalo de confianza 95\% } \\
\cline { 2 - 4 } & & Inferior & Superior \\
\hline Sensibilidad & $83,72 \%$ & $75,96 \%$ & $89,42 \%$ \\
Especificidad & $88,78 \%$ & $83,45 \%$ & $92,61 \%$ \\
Valor Predictivo Positivo & $82,44 \%$ & $74,61 \%$ & $88,32 \%$ \\
Valor Predictivo Negativo & $89,66 \%$ & $84,42 \%$ & $93,33 \%$ \\
\hline
\end{tabular}

\section{DISCUSIÓN}

En este estudio se encontró que la sensibilidad de la prueba rápida comparada con el TPHA fue de $83,72 \%$ (IC95\%: $75,96-89,42 \%$ ) y la especificidad fue de $88,78 \%$ (IC95\%: $83,45-92,61 \%)$ siendo los valores predictivos positivos y negativos de $82,44 \%$ (IC95\%:74,61\%-88,32\%) y $89,66 \%$ (IC del $95 \% 84.42 \%-93.33 \%$ ) en un total de 334 muestras de mujeres puérperas. En un estudio realizado en California, Estados Unidos con 948 pacientes de ambos sexos con riesgo de ITS pero baja prevalencia de sífilis, la sensibilidad de la prueba rápida con el TPHA fue de 52,7\%(IC95\%: 42,1-63,1\%) y en nuestro estudio fue más alta, de $83,72 \%(10)$. Sin embargo, la especificidad obtenida en nuestro estudio fue de $88,78 \%$ más baja que la obtenida en el citado estudio98.7\% (IC95\%: 97,5-99,4\%).Aunque el TPHA generalmente permanece detectable de por vida después de la infección, trabajos previos han demostrado una disminución de la concentración de TPHA después del tratamiento pudiendo haber descendido en los casos tratados por debajo del nivel de detección de la prueba rápida, pero sigue siendo detectable con TPHA $(10,11)$.

Una limitación fue que no contábamos con información si los casos positivos de sífilis eran nuevos o tratados, siendo posible que algunos falsos negativos con la prueba rápida sean casos tratados. En otro estudio en Francia de 100 pacientes adultos con diversos estadios 
de sífilis la sensibilidad de las pruebas rápidas comparadas con ELISA para anticuerpos treponémicos fue por encima de $90 \%$, así como la especificidad. Al considerar los débiles reactivos, la especificidad bajo al 88,2\%.(11) En un estudio en Colombia, realizado en mujeres sexualmente activas de 14 a 49 años los valores del SD Bioline para sífilis fueron: Sensibilidad: 91.6 (IC95 \%: 71.8 a 100) y Especificidad 98.8 (IC95 \%: 98.2 a 99.4) en comparación con TPHA (12). Asimismo, en otro estudio realizado en India en pacientes de ambos sexos y todas las edades se reportaron sensibilidad y especificidad de $92.86 \%$ y $98.28 \%$, respectivamente, para SD BIOLINE Sífilis 3.0 , en comparación con TPHA (13).Sensibilidad y especificidad mayores a $98 \%$ comparando SD Bioline Syphilis 3.0 y TPHA también han sido reportadas en un estudio en Corea(14). Con esta misma tira rápida y en un estudio en África en 534 mujeres embarazadas con varios estadios de Sífilis la sensibilidad fue de91.9\% (IC95 \% :89.2-94.1)y especificidad 99.5\% (IC95\%:99.2-99.7) (15) y con otras marcas de tiras rápidas y en un total de 1325 mujeres de 15-54 años se obtuvieron sensibilidad y especificidad de $75 \%$ y $95 \%$ respectivamente respecto al TPHA(16). En nuestro estudio fueron positivas las muestras que tenían desde una coloración débil hasta fuerte en la línea de testeo de acuerdo a las indicaciones del fabricante y la sensibilidad obtenida de $84 \%$ concuerda con lo aceptado por un estudio de pruebas rápidas de sífilis evaluados por la Organización Mundial de la Salud (OMS), que muestra como rango aceptable del 84 a 97\% comparando las pruebas rápidas con el TPHA(17). En cuanto a la especificidad encontrada, de $88,78 \%$ comparada con TPHA es también aceptable con respecto a lo publicado con la OMS, que prevé especificidades de $84 \%$ en adelante $(17,18)$.Se han encontrado mejores desempeños como en un estudio realizado en Bolivia en el cual la sensibilidad y especificidad de las pruebas rápidas encontradas comparadas con TPHA fue de 97,6 y 97,3 en 8924 embarazadas, sin conocer status de estado inmunológico contra la sífilis(18).

En cuanto a la distribución por edad se observa una mayor cantidad de casos positivos de sífilis entre mujeres de 13 y 30 años con un porcentaje mayor de puérperas adolescentes (19\%) que mujeres entre 31 a 35 años (15\%) y entre 36 y 40 años (11\%)lo que también describe un estudio en adolescentes paraguayas (19).

El uso de pruebas rápidas es muy importante en la estrategia de la OMS para la eliminación global de la sífilis congénita y de la transmisión de madre a hijo tanto de la sífilis como del VIH, porque permiten detección y tratamiento en la misma visita sobre el terreno o clínicas periféricas alejadas de laboratorios $(17,20)$. Con base en la epidemiologia de la sífilis gestacional se concluye que el análisis de sífilis en puérperas y/o parturientas o puerperio inmediato puede usarse para obtener una estimación de la magnitud de sífilis congénita, que en nuestro país es de 8,3 por 100.000 nacidos vivos, superior a varios países de la región y al 0,5\% establecido como indicador por la OPS por para la medición del impacto establecido por la OPS en el plan de acción para eliminar la sífilis congénita (7).Una desventaja de las tiras rápidas sería no poder distinguir entre infección activa y anterior, sin embargo, se consideran útiles en tamizaje de poblaciones de gestantes para prevenir la sífilis congénita, o en grupos en riesgo como en profesionales del sexo, hombres que mantienen sexo con hombres, individuos con ITS o con vulnerabilidad a estas enfermedades y consumidores de drogas inyectables, en los cuales el beneficio de administrar el tratamiento a todos los positivos supera ampliamente al daño potencial del margen de tratamiento innecesario, teniendo en cuenta las secuelas potencialmente graves de la enfermedad y el mayor riesgo de transmisión de VIH. Sin embargo, estas pruebas sólo detectan la presencia de anticuerpos treponémicos, por lo que no puede distinguir entre los casos activos y casos históricos, tratados (1).

En nuestro estudio, la sensibilidad y especificidad habrían sido más precisas si la muestra hubiese incluido más casos positivos de sífilis, pero también es importante determinar la exactitud de la prueba en poblaciones de baja prevalencia si la prueba rápida va a ser utilizada en todos los tipos de poblaciones $(10,11)$.

Existen políticas públicas en nuestro país para prevenir la sífilis congénita y neonatal como la descrita en la Resolución S.G No 147 del MSP y BS que crea una Comisión Técnica de la Maternidad Segura y Mortalidad Neonatal, con orientaciones específicas y acciones inmediatas para mejorar la salud materna y acelerar la reducción de la morbimortalidad materna y neonatal (21).Previa a esta resolución se estableció la Norma clínica para la eliminación de la Transmisión Materno Infantil del VIH y de la Sífilis Congénita que por resolución SG No 246 del 12 de Abril de 2011 dispone su aplicación obligatoria en todo el territorio nacional. Se establece en la misma que toda mujer embarazada debe ser analizada serológicamente con una prueba rápida de sífilis en la primera consulta prenatal, 
debiendo hacerse una segunda prueba serológica entre las 28 y 32 semanas y en el momento del parto o puerperio inmediato una tercera serología y eventualmente cuando sea atendida para curetaje por aborto o mortinato (22). Es obligatorio el desarrollo del sistema de atención oportuna con procedimientos que permitan detectar inconvenientes u obstáculos y ayuden a mejorar la eficiencia de trabajo hacia la prevención de la transmisión perinatal y sífilis congénita con fortalecimiento de los centros de atención de salud y de los laboratorios clínicos (23-25). La prueba rápida realizada siguiendo los protocolos del fabricante es una herramienta de detección para las poblaciones de bajo riesgo. Esto, combinado con pruebas en el lugar y en el mismo día de tratamiento podría ampliar los programas de detección de sífilis prenatal y en puérperas en comunidades distantes que se caracterizan por la dificultad de acceso a los servicios de atención prenatal y visitas de seguimiento clínico poco frecuentes y en sistemas de salud que cuentan con pocos recursos $(4,26,27)$. Aunque las pruebas y el tratamiento de la sífilis en el embarazo deben estar universalmente disponibles, un estudio de la OMS revela que más de la mitad de los embarazos en mujeres con sífilis dará lugar a un resultado adverso. Esta es una carga evitable en las madres, las familias y los sistemas de salud que pone de relieve la necesidad de dar prioridad a los esfuerzos mundiales para eliminar la transmisión de madre a hijo de la sífilis $(28,29)$.

\section{REFERENCIAS BIBLIOGRÁFICAS}

1. Castañeda BC. Costo efectividad del uso de pruebas treponémicas rápidas para el tratamiento temprano de la Sífilis Gestacional en pacientes subsidiadas y no afiliadas al Sistema General de Seguridad Social en Salud en Bogotá. Tesis de Grado, Universidad Nacional de Colombia, Facultad de Medicina, Maestría en Epidemiología Clínica. Bogotá, Colombia, 2011. Disponible en:

http://www.bdigital.unal.edu.co/6405/1/598 168.2012.pdf

2. Villazón $N$, Conde $C$, Juárez $L$, Uribe $F$. Evaluation of a rapid diagnostic test to assess the prevalence of maternal syphilis in Bolivia. Rev. méd. Chile 2009 April;137(4):515-21.

3. Janier $M$, Hegyi V, Dupin N, Unemo $M$, Tiplica G, Patel R et al. European guideline on the management of syphilis. J Eur Acad Dermatol Venereol. 2014;28(12):1581-93.

4. ÁngelE, González MP, Núñez L, Pacheco J, Tolosa JE, Díaz $\mathrm{L}$ et al. Frecuencia de infecciones del tracto genital femenino en mujeres sintomáticas y uso de pruebas rápidas para su diagnóstico en dos poblaciones de Bogotá (Colombia) 2008: Estudio piloto. Rev Colomb Obstet Ginecol. 2010;61(3):220-30.

5. Pope V, Fears MB, Morrill WE, Castro A, Kikkert SE. Comparison of the Serodia Treponema pallidum Particle Agglutination, Captia Syphilis-G, and SpiroTek Reagin II Tests with Standard Test Techniques for Diagnosis of Syphilis. J Clin Microbiol. 2000; 38(7):2543-5.

6. Omoding $D$, Katawera $V$, Siedner $M$, Yap Boum I. Evaluation of the SD BIOLINE HIV/syphilis Duo assay at a rural health center in Southwestern Uganda. BMC Research Notes. 2014;7(1):746-9.

7. Ministerio de Salud Pública y Bienestar Social. Programa Nacional de Control del VIH/ITS. Estudio de prevalencia de sífilis y VIH en parturientas y puérperas en el
Paraguay 2012 [Internet]. PRONASIDA; 2012. [Citado 1 mar 2015]Disponibleen: http://www.pronasida.gov.py/images/docum entos/material_educativo/inf_est_puerperas. pdf.

8. Mabey DC, Sollis KA, Kelly HA, Benzaken AS, Bitarakwate $\mathrm{E}$, Changalucha J et al. Point-ofCare Tests to Strengthen Health Systems and Save Newborn Lives: The Case of Syphilis. PLoSMed. 2012;9(6):e1001233. Doi:10.1371/journal.pmed.1001233

9. HernándezM, HernándezB, UribeF, Juárez L, CondeCJ. Maternal and congenital syphilis in two Mexican hospitals: Evaluation of a rapid diagnostic test. Rev Invest Clin. 2006;58(2):119-25.

10. Hess KL, Fisher DG, Reynolds GL. Sensitivity and specificity of point-of-care rapid combination syphilis-HIV-HCV tests. PLoS One. 2014;9(11):e112190.Doi: 10.1371/journal.pone.0112190.

11. Guinard J, Prazuck T, Péré $H$, Poirier $C$, LeGoff J, Boedec E. et al. Usefulness in clinical practice of a point-of-care rapid test for simultaneous detection of non treponemal and Treponema pallidum-specific antibodies in patients suffering from documented syphilis. Int J STD AIDS. 2013;24(12):944-50.

Doi:

10.1177/0956462413487328.

12. Nuñez L, Moyano L, Gaitan $H$, Angel E, Ruiz A, Gonzalez $P$ et al. Diagnostic accuracy of rapid tests for sexually transmitted infections in symptomatic women. Sex TransmInfect.2016;92(1):24-8. Doi: 10.1136/sextrans-2014-051891.

13. Mehra B, Bhattar S, Saxena S, Rawat D, Bhalla P. Evaluation of SD BIOLINE Syphilis 3.0 for Rapid Diagnosis of Syphilis: Report from a Regional Sexually Transmitted Infection Reference Laboratory in North India. J Lab Physicians.2016;8(1):36-40. Doi: $10.4103 / 0974-2727.176239$.

14. Lee JH, Lim CS, Lee MG, Kim HS. Evaluation of a Rapid Immunochromatographic 
Treponemal Antibody Test Comparing the Treponema Pallidum Particle Agglutination Assay. J Clin Lab Anal. 2015; 29(5):383-6. Doi: $10.1002 /$ jcla.21783.

15. Montoya PJ, Lukehart SA, Brentlinger PE, Blanco AJ, Floriano F, Sairosse J, Gloyd S. Comparison of the diagnostic accuracy of a rapid immunochromatographic test and the rapid plasma reagin test for antenatal syphilis screening in Mozambique. Bull World Health Organ. 2006;84(2):97-104.

16. West B, Walraven G, Morison L, Brouwers J, Bailey $R$. Performance of the rapid plasma reagin and the rapid syphilis screening tests in the diagnosis of syphilis in field conditions in rural Africa. Sex Transm Infect. $2002 ; 78(4): 282-5$.

17. Iniciativa para el Diagnóstico de Enfermedades de Transmisión Sexual (IMDETS), Programa Especial para la Investigación y Capacitación en Enfermedades Tropicales (TDR) patrocinado por UNICEF/PNUD/Banco Mundial/OMS Número de páginas: 28 Fecha de publicación: 2007 disponible en http://www.who.int/reproductivehealth/publi cations/rtis/TDR_SDI_06_1/es/

18. Tinajeros F, Grossman D, Richmond K, Steele M, Garcia SG, Zegarra L, Revollo R. Diagnostic accuracy of a point-of-care syphilis test when used among pregnant women in Bolivia. Sex Transm Infect. 2006;82Suppl5:v17-21.

19. Rodríguez $M$, Insaurralde $A$, Páez $M$, Mendoza $L$, Castro A, Giménez G. Conocimientos, actitudes y prácticas sobre sífilis materna: adolescentes embarazadas. Hospitales de referencia en Paraguay. 2008. Mem. Inst. Investig. Cienc. Salud. 2012;10(1):76-84.

20. World Health Organization. The Sexually Transmitted Diseases Diagnostics Initiative (SDI). Special Programmefor Research and Training in Tropical Diseases (TDR), Geneva, http://www.who.int/tdr/publications/docume nts/sdi.pdf

21. RESOLUCION SG No 407 DEL Ministerio de salud pública y Bienestar Social .3 de mayo de 2013 Por la cual se crea la comisión técnica de la maternidad segura y mortalidad neonatal disponible en http://www.mspbs.gov.py/v2/documentacio n.php?\&page $=4$
22. Guía Clínica para la eliminación de la transmisión Materno Infantil del VIH y de la Sífilis Congénita en el Paraguay 2012 -2013 Proyecto VIH /SIDA/ITS Beneficiario Principal: Centro de Información y recursos para el desarrollo, CIRD Sub beneficiario Programa Nacional de Control de SIDA IBSN 978-999953-32-62-4.

23. San MiguelC, Vera $E$, Fanego $H$. Seroprevalencia de Sífilis en embarazadas Alto Parana-2008. Rev. Inst. Med. Trop. 2010;5(1):7-13.

24. Arbo A. Sífilis: situación alarmante en el Paraguay. Rev. Inst. Med. Trop. 2010;5(1):4-5.

25. Caballero S, Valenzuela A, Evers S, Mendoza L. Infecciones de transmisión sexual en mujeres de edad fértil usuarias del Hospital Regional de Villa Hayes, Paraguay. Mem. Inst.Investig. Cienc. Salud. 2015;13(2):3949.

26. Jafari $Y$, Peeling RW, Shivkumar $S$, Claessens C, Joseph L, Pai NP. Are Treponema Pallidum specific rapid and pointof-care tests for syphilis accurate enough for screening in resource limited settings? Evidence from a meta-analysis. PLoS One.2013;8(2):e54695.

10.1371/journal.pone.0054695.

27. Benzaken AS, Sabidó M, Galban E, Pedroza $\mathrm{V}$, Araújo AJ, Peeling RWet al. Field performance of a rapid point-of-care diagnostic test for antenatal syphilis screening in the Amazon region, Brazil. Int J STD AIDS. 2011;22(1):15-8. Doi: 10.1258/ijsa.2010.010145.

28. Gomez G, Kamb M, Newman L, Mark J, Broutet N, Hawkes S. Untreated maternal syphilis and adverse outcomes of pregnancy: a systematic review and meta-analysis. Bull World Health Organ. 2013;91(3):217-26. Doi: 10.2471/BLT.12.107623

29. Rodrigues CS, Guimarães MD, Grupo Nacional de Estudio sobre Sífilis Congénita. Syphilis positivity in puerperal women: still a challenge in Brazil. Rev PanamSaludPublica.2004;16(3):168-75. 